\title{
A condition for blow-up solutions to discrete $p$-Laplacian parabolic equations under the mixed boundary conditions on networks
}

\author{
Soon-Yeong Chung ${ }^{1,2^{*}}$, Min-Jun Choi ${ }^{2,3}$ and Jaeho Hwang ${ }^{2}$
}

\author{
"Correspondence: \\ sychung@sogang.ac.kr \\ ${ }^{1}$ National Institute for Mathematical \\ Sciences, Daejeon, Republic of \\ Korea \\ ${ }^{2}$ Department of Mathematics, \\ Sogang University, Seoul, Republic \\ of Korea \\ Full list of author information is \\ available at the end of the article
}
Abstract
In this paper, we investigate the condition$$
\left(C_{p}\right) \quad \alpha \int_{0}^{u} f(s) d s \leq u f(u)+\beta u^{p}+\gamma, \quad u>0
$$

for some $\alpha>2, \gamma>0$, and $0 \leq \beta \leq \frac{(\alpha-p) \lambda_{p, 0}}{p}$, where $p>1$, and $\lambda_{p, 0}$ is the first eigenvalue of the discrete $p$-Laplacian $\Delta_{p, \omega}$. Using this condition, we obtain blow-up solutions to discrete $p$-Laplacian parabolic equations

$$
\begin{cases}u_{t}(x, t)=\Delta_{p, \omega} u(x, t)+f(u(x, t)), & (x, t) \in S \times(0,+\infty), \\ \mu(z) \frac{\partial u}{\partial p n}(x, t)+\sigma(z)|u(x, t)|^{p-2} u(x, t)=0, & (x, t) \in \partial S \times[0,+\infty), \\ u(x, 0)=u_{0} \geq 0 \quad \text { (nontrivial), } & x \in S,\end{cases}
$$

on a discrete network $S$, where $\frac{\partial u}{\partial p n}$ denotes the discrete $p$-normal derivative. Here $\mu$ and $\sigma$ are nonnegative functions on the boundary $\partial S$ of $S$ with $\mu(z)+\sigma(z)>0$, $z \in \partial S$. In fact, we will see that condition $\left(C_{p}\right)$ improves the conditions known so far.

MSC: 39A 12; 35F31; 35K91; 35K57

Keywords: Discrete $p$-Laplacian; Semilinear parabolic equation; Blow-up

\section{Introduction}

These days the discrete version of differential equations has attracted attention of many researchers. In particular, the $p$-Laplacian $\Delta_{p, \omega}$ on networks (or weighted graphs) is used to observe various social and scientific phenomena (see [1-3] and references therein), which can be modeled by the discrete $p$-Laplacian parabolic equations

$$
u_{t}=\sum_{y \in \bar{S}}|u(y, t)-u(x, t)|^{p-2}[u(y, t)-u(x, t)] \omega(x, y)+f(u)
$$

with some boundary and initial conditions, where $p>1$. Here $\bar{S}$ is the set of chemicals or networks, and $\omega$ is a weight function on $\bar{S}$.

(c) The Author(s) 2019. This article is distributed under the terms of the Creative Commons Attribution 4.0 International License (http://creativecommons.org/licenses/by/4.0/), which permits unrestricted use, distribution, and reproduction in any medium, provided you give appropriate credit to the original author(s) and the source, provide a link to the Creative Commons license, and indicate if changes were made. 
Especially, many authors studied blow-up solutions for the reaction-diffusion equations, which contain $p$-Laplacian, Laplacian, and so on, in continuous and discrete analogues. For example, in 1973, Levine [4] considered formal parabolic equations of the form

$$
\left\{\begin{array}{l}
P \frac{d u}{d t}=-A(t) u+f(u(t)), \quad t \in[0,+\infty) \\
u(0)=u_{0}
\end{array}\right.
$$

where $P$ and $A(t)$ are positive linear operators defined on a dense subdomain $D$ of a real or complex Hilbert space $H$. Here he first introduced the "concavity method" to obtained the blow-up solutions under abstract conditions

$$
2(\alpha+1) F(x) \leq(x, f(x)), \quad F\left(u_{0}(x)\right)>\frac{1}{2}\left(u_{0}(x), A u_{0}(x)\right)
$$

for $x \in D$, where $F(x)=\int_{0}^{1}(f(\rho x), x) d \rho$.

After this, Philippin and Proytcheva [5] have applied this method to the equations

$$
u_{t}=\Delta u+f(u) \text { in } \Omega \times(0,+\infty)
$$

and obtained a blow-up solution under the Dirichlet boundary condition by using the condition

$$
(A): \quad(2+\epsilon) F(u) \leq u f(u), \quad u>0,
$$

for some $\epsilon>0$ and the initial data $u_{0}$ satisfying

$$
-\frac{1}{2} \int_{\Omega}\left|\nabla u_{0}(x)\right|^{2} d x+\int_{\Omega} F\left(u_{0}(x)\right) d x>0 .
$$

For the $p$-Laplace operator, Messaoudi [6] obtained the blow-up solutions to the equation

$$
u_{t}=\operatorname{div}\left(|\nabla u|^{p-2} \nabla u\right)+f(u) \quad \text { in } \Omega \times(0,+\infty)
$$

under the Dirichlet boundary condition by using the condition

$$
\left(A_{p}\right): \quad(p+\epsilon) F(u) \leq u f(u), \quad u>0,
$$

for some $\epsilon>0$ and the initial data $u_{0}$ satisfying

$$
-\frac{1}{p} \int_{\Omega}\left|\nabla u_{0}(x)\right|^{p} d x+\int_{\Omega} F\left(u_{0}(x)\right) d x>0 .
$$

Besides, Junning [7] obtained the blow-up solutions to the equation

$$
u_{t}=\operatorname{div}\left(|\nabla u|^{p-2} \nabla u\right)+f(u) \quad \text { in } \Omega \times(0,+\infty)
$$


under the Dirichlet boundary condition by using the condition

$$
\left(A_{p}^{\prime}\right): \quad p F(u) \leq u f(u), \quad u>0 .
$$

Here the initial data $u_{0}$ satisfies

$$
-\frac{1}{p} \int_{\Omega}\left|\nabla u_{0}(x)\right|^{p} d x+\int_{\Omega} F\left(u_{0}(x)\right) d x>\frac{4(p-1)}{T(p-2)^{2} p} \int_{\Omega} u_{0}^{2}(x) d x .
$$

Recently, Ding and $\mathrm{Hu}[8]$ adopted condition $(A)$ to obtain the blow-up solutions to the equation

$$
(g(u))_{t}=\nabla \cdot\left(\rho\left(|\nabla u|^{2}\right) \nabla u\right)+k(t) f(u)
$$

with the nonnegative initial value and the null Dirichlet boundary condition.

On the other hand, condition (A) was relaxed by Bandle and Brunner [9] as follows:

$$
(B): \quad(2+\epsilon) F(u) \leq u f(u)+\gamma, \quad u>0,
$$

for some $\epsilon>0$. Also, the initial data $u_{0}$ satisfies

$$
-\frac{1}{2} \int_{\Omega}\left|\nabla u_{0}(x)\right|^{2} d x+\int_{\Omega}\left[F\left(u_{0}\right)-\gamma\right] d x>0
$$

for some $\epsilon>0$ and $\gamma>0$.

Finally, condition $(B)$ was improved by Chung and Choi [10] with the discrete analogue. They obtained the blow-up solutions to the equation

$$
u_{t}=\Delta_{\omega} u(x, t)+f(u) \quad \text { in } S \times(0,+\infty)
$$

under the Dirichlet boundary condition by using the condition

$$
(C): \quad(2+\epsilon) F(u) \leq u f(u)+u^{2}+\gamma, \quad u>0,
$$

for some $\epsilon>0,0<\beta \leq \frac{\epsilon \lambda_{0}}{2}$, and $\gamma>0$ and the initial data $u_{0}$ satisfying

$$
-\frac{1}{2} \sum_{x \in \bar{S}}\left|u_{0}(x)-u_{0}(y)\right|^{2} \omega(x, y)+\sum_{x \in \bar{S}}\left[F\left(u_{0}\right)-\gamma\right] d x>0 .
$$

Here $\lambda_{0}$ is the first eigenvalue for the discrete Laplace operator $\Delta_{\omega}$.

In 2018, Chung and Choi [11] refines condition (C) in continuous analogue. For $p \geq 2$, they obtained the blow-up solutions to the equation

$$
u_{t}=\operatorname{div}\left(|\nabla u|^{p-2} \nabla u\right)+f(u) \quad \text { in } \Omega \times(0,+\infty)
$$

under the Dirichlet boundary condition by using the condition

$$
\left(C_{p}\right): \quad(p+\epsilon) F(u) \leq u f(u)+\beta u^{p}+\gamma, \quad u>0,
$$


for some $\epsilon>0,0<\beta \leq \frac{\epsilon \lambda_{p, 0}}{p}$, and $\gamma>0$. Here the initial data $u_{0}$ satisfies

$$
-\frac{1}{p} \int_{\Omega}\left|\nabla u_{0}(x)\right|^{p} d x+\int_{\Omega} F\left(u_{0}(x)\right) d x>0 .
$$

Here $\lambda_{p, 0}$ is the first eigenvalue for the $p$-Laplace operator.

It is clear that conditions $(A),\left(A_{p}\right),(B)$, and $\left(B_{p}\right)$ are independent of the eigenvalue of the Laplace operator, and conditions $(C)$ and $\left(C_{p}\right)$ depend on the eigenvalue. As a matter of fact, it is known that the first eigenvalue for the $p$-Laplace operator depends not only on the domain but also on the boundary conditions (see [12]).

Motivated by the works mentioned, we study the blow-up solutions to the following discrete $p$-Laplacian parabolic equations:

$$
\begin{cases}u_{t}(x, t)=\Delta_{p, \omega} u(x, t)+f(u(x, t)), & (x, t) \in S \times(0,+\infty), \\ B[u]=0 & \text { on } \partial S \times[0,+\infty), \\ u(x, 0)=u_{0}(x) \geq 0, & x \in \bar{S},\end{cases}
$$

where $p>1, f$ is a nonnegative locally Lipschitz continuous function on $\mathbb{R}$, and $B[u]=0$ on $\partial S \times[0,+\infty)$ stands for the boundary condition

$$
\mu(z) \frac{\partial u}{\partial_{p} n}(z, t)+\sigma(z)|u(z, t)|^{p-2} u(z, t), \quad(z, t) \in \partial S \times[0,+\infty) .
$$

Here $\mu, \sigma: \partial S \rightarrow[0,+\infty)$ are functions such that $\mu(z)+\sigma(z)>0, z \in \partial S$, and $\frac{\partial u}{\partial p n}$ denotes the discrete $p$-normal derivative (introduced in Sect. 1). It is easy to see that this boundary value problem includes various boundary value problems such as the Dirichlet boundary, Neumann boundary, and Robin boundary problems. Note that one of advantages of our result is a unified approach.

To obtain the blow-up solutions to equation (2), we introduce the following condition:

For $p>1$,

$$
\left(C_{p}\right): \quad \alpha F(u) \leq u f(u)+u^{p}+\gamma, \quad u>0,
$$

for some $\alpha>2,0 \leq \beta \leq \frac{(\alpha-p) \lambda_{p, 0}}{p}$, and $\gamma \geq 0$.

We discuss condition $\left(C_{p}\right)$ in Section 3 to understand the constants $\alpha, \beta$, and $\gamma$ with respect to the boundary condition $B[u]=0$ and the parameter $p>1$, which are crucial points of our results.

It is worth noting that we obtained the blow-up solutions to equation (2) in the case $p>1$, not in the case $p \geq 2$. In fact, there are interesting results in the case $1<p<2$ with respect to blow-up property (see [13-15]). Therefore we expect that under condition $\left(C_{p}\right)$, more interesting results can be obtained even in the continuous case, which will be our forthcoming work.

We organize this paper as follows. In Sect. 1, we briefly introduce the preliminary concepts on networks and comparison principles. Section 2 is the main section devoted to blow-up solutions using the concavity method with condition $\left(C_{p}\right)$. Finally, in Sect. 3, we discuss condition $\left(C_{p}\right)$, comparing it with conditions $\left(A_{p}\right)$ and $\left(B_{p}\right)$, together with the condition $B(0)>0$, the parameter $p>1$, and the initial data condition. 


\section{Preliminaries and discrete comparison principles}

In this section, we start with the theoretic graph notions frequently used throughout this paper. For more detailed information on notations, notions, and conventions, we refer the reader to [16].

\section{Definition 1.1}

(i) A graph $G=G(V, E)$ is a finite set $V$ of vertices with a set $E$ of edges (two-element subsets of $V$ ). Conventionally used, we denote by $x \in V$ or $x \in G$ the fact that $x$ is a vertex in $G$.

(ii) A graph $G$ is called simple if it has neither multiple edges nor loops.

(iii) $G$ is called connected if for all vertices $x$ and $y$, there exists a sequence of vertices $x=x_{0}, x_{1}, \ldots, x_{n-1}, x_{n}=y$ such that $x_{j-1}$ and $x_{j}$ are connected by an edge for $j=1, \ldots, n$ (called adjacent).

(iv) A graph $G^{\prime}=G^{\prime}\left(V^{\prime}, E^{\prime}\right)$ is called a subgraph of $G(V, E)$ if $V^{\prime} \subset V$ and $E^{\prime} \subset E$. In this case, $G$ is a host graph of $G^{\prime}$. If $E^{\prime}$ consists of all the edges from $E$ that connect the vertices of $V^{\prime}$ in its host graph $G$, then $G^{\prime}$ is called an induced subgraph.

Definition 1.2 For an induced subgraph $S$ of a graph $G=G(V, E)$, the (vertex) boundary $\partial S$ of $S$ is defined as

$\partial S:=\{z \in V \backslash S \mid z \sim y$ for some $y \in S\}$.

Here, $x \sim y$ means that two vertices $x$ and $y$ are connected (adjacent) by an edge in $E$. Throughout this paper, the subgraph $S$ is assumed to be induced, simple, and connected. Also, we denote by $\bar{S}$ the graph with vertices and edges in $S \cup \partial S$. We note that by definition the set $\bar{S}$ is an induced subgraph of $G$.

Definition 1.3 A weight on a graph $G$ is a symmetric function $\omega: V \times V \rightarrow[0,+\infty)$ satisfying the following:

(i) $\omega(x, x)=0, x \in V$,

(ii) $\omega(x, y)=\omega(y, x)$ if $x \sim y$,

(iii) $\omega(x, y)>0$ if and only if $\{x, y\} \in E$,

and a graph $G$ with weight $\omega$ is called a network.

Definition 1.4 The degree $d_{\omega} x$ of a vertex $x$ in a network $S$ (with boundary $\partial S$ ) is defined as

$$
d_{\omega} x:=\sum_{y \in \bar{S}} \omega(x, y)
$$

Definition 1.5 For $p>1$ and a function $u: \bar{S} \rightarrow \mathbb{R}$, the discrete $p$-Laplacian $\Delta_{p, \omega}$ on $S$ is defined by

$$
\Delta_{p, \omega} u(x):=\sum_{y \in \bar{S}}|u(y)-u(x)|^{p-2}[u(y)-u(x)] \omega(x, y)
$$

for $x \in S$. 
Definition 1.6 For $p>1$ and a function $u: \bar{S} \rightarrow \mathbb{R}$, the discrete $p$-normal derivative $\frac{\partial u}{\partial_{p} n}$ on $\partial S$ is defined by

$$
\frac{\partial u}{\partial_{p} n}(z):=\sum_{x \in S}|u(z)-u(x)|^{p-2}[u(z)-u(x)] \omega(x, z)
$$

for $z \in \partial S$.

The following two lemmas are used throughout this paper.

Lemma 1.7 (See [17]) Let $p>1$. For functions $f, g: \bar{S} \rightarrow \mathbb{R}$, the discrete $p$-Laplacian $\Delta_{p, \omega}$ satisfies

$$
\begin{aligned}
2 \sum_{x \in \bar{S}} g(x) & {\left[-\Delta_{p, \omega} f(x)\right] } \\
& =\sum_{x, y \in \bar{S}}|f(y)-f(x)|^{p-2}[f(y)-f(x)][g(y)-g(x)] \omega(x, y) .
\end{aligned}
$$

In particular, in the case $g=f$, we have

$$
2 \sum_{x \in \bar{S}} f(x)\left[-\Delta_{p, \omega} f(x)\right]=\sum_{x, y \in \bar{S}}|f(x)-f(y)|^{p} \omega(x, y) .
$$

Lemma 1.8 (See [12]) For $p>1$, there exist $\lambda_{p, 0}>0$ and a function $\phi_{0}(x)>0, x \in S \cup \Gamma$, such that

$$
\begin{cases}-\Delta_{p, \omega} \phi_{0}(x)=\lambda_{p, 0}\left|\phi_{0}(x)\right|^{p-2} \phi_{0}(x), & x \in S, \\ B\left[\phi_{0}\right]=0 & \text { on } \partial S\end{cases}
$$

where $B\left[\phi_{0}\right]$ on $\partial S$ stands for

$$
\mu(z) \frac{\partial \phi_{0}}{\partial_{p} n}(z)+\sigma(z)\left|\phi_{0}(z)\right|^{p-2} \phi_{0}(z), \quad z \in \partial S .
$$

Here $\Gamma:=\{z \in \partial S \mid \mu(z)>0\}$, and $\mu, \sigma: \partial S \rightarrow[0,+\infty)$ are functions such that $\mu(z)+\sigma(z)>0$ for $z \in \partial S$. Moreover, $\lambda_{p, 0}$ is given by

$$
\lambda_{p, 0}=\min _{u \in \mathcal{A}, u \neq 0} \frac{\frac{1}{2} \sum_{x, y \in \bar{S}}|u(x)-u(y)|^{p} \omega(x, y)+\sum_{z \in \Gamma} \frac{\sigma(z)}{\mu(z)}|u(z)|^{p}}{\sum_{x \in S}|u(x)|^{p}},
$$

where $\mathcal{A}:=\{u: \bar{S} \rightarrow \mathbb{R} \mid u \neq \equiv$ in $S, u=0$ on $\partial S \backslash \Gamma\}$.

The number $\lambda_{p, 0}$ is called the first eigenvalue of $\Delta_{p, \omega}$ on a network $\bar{S}$ with corresponding eigenfunction $\phi_{0}$ (see [18] and [19] for the spectral theory of the Laplacian operators). In fact, we note that if $\Gamma$ is the empty set, then $\sum_{z \in \Gamma} \frac{\sigma(z)}{\mu(z)}|u(z)|^{p}$ is 0 .

Remark 1.9 It is clear that the first eigenvalue $\lambda_{p, 0}$ is nonnegative. Moreover, we note here that the first eigenvalue $\lambda_{p, 0}$ satisfies the following statements: 
(i) If $\sigma \equiv 0$, then $\lambda_{p, 0}=0$.

(ii) If $\sigma \not \equiv 0$, then $\lambda_{p, 0}>0$.

We now discuss the local existence of a solution to equation (2). To discuss the local existence, we would like to investigate the relationship between the boundary condition $B[u]=0$ and the initial data $u_{0}$.

Remark 1.10 Consider the function $\psi: \mathbb{R} \rightarrow \mathbb{R}$ defined as

$$
\psi(\gamma):=\sum_{x \in S}|\gamma-u(x, t)|^{p-2}[\gamma-u(x, t)] a(x)+b|\gamma|^{p-2} \gamma
$$

where $a(x) \geq 0$ for $x \in S$, and $b \geq 0$ with $a(x)+b>0$ for some $x \in S$. Then it is easy to see that $\psi$ is a continuous function that is strictly increasing and bijective on $\mathbb{R}$. Therefore there exists a unique $\rho \in \mathbb{R}$ such that $\psi(\rho)=0$. It means that for all $z \in \partial S$, we can uniquely define the value of $u(z, 0)$ according to the given boundary condition $B[u]=0$ and initial data $u_{0}$, that is, for every $z \in \partial S, u(z, 0)$ is determined such that

$$
\mu(z) \frac{\partial u}{\partial_{p} n}(z, 0)+\sigma(z)|u(z, 0)|^{p-2} u(z, 0)=0, \quad z \in \partial S
$$

where $\mu, \sigma: \partial S \rightarrow[0,+\infty)$ are given functions with $\mu(z)+\sigma(z)>0$ for all $z \in \partial S$. Therefore we have a compatible condition such that the initial data $u_{0}$ satisfies

$$
\mu(z) \frac{\partial u_{0}}{\partial_{p} n}(z)+\sigma(z)\left|u_{0}(z)\right|^{p-2} u_{0}(z)=0, \quad z \in \partial S .
$$

We will prove the existence of the solution to equation (2) using the Schauder fixed point theorem. For this reason, we first define the set $C(S \times I)$ for a compact interval $I$ :

$$
C(S \times I):=\{u: S \times I \rightarrow \mathbb{R} \mid u(x, \cdot) \in C(I) \text { for each } x \in S\}
$$

Also, we need the following modified version of the Arzelà-Ascoli theorem.

Lemma 1.11 (Modified version of the Arzelà-Ascoli theorem) Let F be a compact subset of $\mathbb{R}$, and let $\bar{S}$ be a network. Consider the Banach space $C(S \times F)$ with the maximum norm $\|u\|_{S, F}:=\max _{x \in S} \max _{t \in F}|u(x, t)|$. Then a subset $A$ of $C(S \times F)$ is relatively compact if $A$ is uniformly bounded on $S \times F$ and equicontinuous on $F$ for each $x \in S$.

Proof This lemma is already proved by Chung and Hwang [14].

Theorem 1.12 (Local existence) There exists $t_{0}>0$ such that equation (2) admits at least one bounded solution $u$ such that $u(x, \cdot)$ is continuous on $\left[0, t_{0}\right]$ and differentiable in $\left(0, t_{0}\right)$ for each $x \in \bar{S}$.

Proof We first start with the Banach space

$$
C\left(S \times\left[0, t_{0}\right]\right):=\left\{u: S \times\left[0, t_{0}\right] \rightarrow \mathbb{R} \mid u(x, \cdot) \in C\left(\left[0, t_{0}\right]\right) \text { for each } x \in S\right\}
$$


with the maximum norm $\|u\|_{S, t_{0}}:=\max _{x \in S} \max _{0 \leq t \leq t_{0}}|u(x, t)|$, where $t_{0} \in \mathbb{R}$ is a positive constant, which will be defined later. Now consider the subspace

$$
B_{t_{0}}:=\left\{u \in C\left(S \times\left[0, t_{0}\right]\right) \mid\|u\|_{S, t_{0}} \leq 2\left\|u_{0}\right\|_{S, t_{0}}\right\}
$$

of a Banach space $C\left(S \times\left[0, t_{0}\right]\right)$. Then it is clear that $B_{t_{0}}$ is convex. To apply the Schauder fixed point theorem, we have to show that $B_{t_{0}}$ is closed. Let $g_{n}$ be a sequence in $B_{t_{0}}$ that converges to $g$. Since the convergence is uniform, $g$ is continuous. Moreover, $\mid\left\|g_{n}\right\|_{S, t_{0}}-$ $\|g\|_{S, t_{0}} \mid \leq\left\|g_{n}-g\right\|_{S, t_{0}}$ implies that $g \in B_{t_{0}}$. Hence $B_{t_{0}}$ is closed.

On the other hand, for every $u \in B_{t_{0}}$, we can uniquely define the value of $u(z, t)$ according to the boundary condition $B[u]=0$ in a similar way to Remark 1.10 , that is, for every $u \in B_{t_{0}}, u(z, t)$ satisfies

$$
\mu(z) \frac{\partial u}{\partial_{p} n}(z, t)+\sigma(z)|u(z, t)|^{p-2} u(z, t)=0, \quad(z, t) \in \partial S \times\left[0, t_{0}\right]
$$

for all $(z, t) \in \partial S \times\left[0, t_{0}\right]$, where $\mu, \sigma: \partial S \rightarrow[0,+\infty)$ are given functions with $\mu(z)+\sigma(z)>0$ for all $z \in \partial S$. Then by the boundary condition it is clear that $u(z, t)$ satisfies $|u(z, t)| \leq$ $\|u\|_{S, t_{0}},(z, t) \in \partial S \times\left[0, t_{0}\right]$.

Let us define the operator $D: B_{t_{0}} \rightarrow B_{t_{0}}$ by

$$
D[u](x, t):=u_{0}(x)+\int_{0}^{t} \Delta_{p, \omega} u(x, s)+f(u(x, s)) d s, \quad(x, t) \in S \times\left[0, t_{0}\right]
$$

where $u_{0}: \bar{S} \rightarrow \mathbb{R}$ is a given function.

Since $f$ is locally Lipschitz continuous on $\mathbb{R}$, there exists $L>0$ such that

$$
|f(a)-f(b)| \leq L|a-b|, \quad a, b \in[-m, m],
$$

where $m=2\left\|u_{0}\right\|_{S, t_{0}}$. Now put

$$
t_{0}:=\frac{\left\|u_{0}\right\|_{S, t_{0}}}{\omega_{0}\left(4\left\|u_{0}\right\|_{S, t_{0}}\right)^{p-1}+4 L\left\|u_{0}\right\|_{S, t_{0}}}
$$

where $\omega_{0}:=\max _{x \in \bar{S}} \sum_{y \in \bar{S}} \omega(x, y)$. Then it is easy to see that the operator $D$ is well-defined. Now we will show that $D$ is continuous. The verification of the continuity is divided into two cases as follows:

(i) $1<p<2$.

For $u$ and $v$ in $B_{t_{0}}$, it follows that

$$
\begin{aligned}
|D[u](x, t)-D[v](x, t)| & \leq\left|\int_{0}^{t_{0}} \sum_{y \in \bar{S}} 2^{2-p}\|u-v\|_{S, t_{0}}^{p-1} \omega(x, y)+L\|u-v\|_{S, t_{0}} d s\right| \\
& \leq t_{0}\left[2^{2-p} \omega_{0}\|u-v\|_{S, t_{0}}^{p-1}+L\|u-v\|_{S, t_{0}}\right] .
\end{aligned}
$$

(ii) $p \geq 2$. 
For $u$ and $v$ in $B_{t_{0}}$, by the mean value theorem we have

$$
\begin{aligned}
& |D[u](x, t)-D[v](x, t)| \\
& \quad \leq\left|\int_{0}^{t_{0}} \sum_{y \in \bar{S}}(p-1)\left\|2 u_{0}\right\|_{S, t_{0}}^{p-2} 2\|u-v\|_{S, t_{0}} \omega(x, y)+L\|u-v\|_{S, t_{0}} d s\right| \\
& \quad \leq\left|\int_{0}^{t_{0}} \sum_{y \in \bar{S}} 2^{2 p-3}(p-1)\left\|u_{0}\right\|_{S, t_{0}}^{p-2}\|u-v\|_{S, t_{0}} \omega(x, y)+L\|u-v\|_{S, t_{0}} d s\right| \\
& \quad \leq t_{0}\left[2^{2 p-3}(p-1)\left\|u_{0}\right\|_{S, t_{0}}^{p-2} \omega_{0}\|u-v\|_{S, t_{0}}+L\|u-v\|_{S, t_{0}}\right] .
\end{aligned}
$$

Consequently, for each $p>1$, we obtain

$$
\|D[u]-D[v]\|_{S, t_{0}} \leq C_{1}\|u-v\|_{S, t_{0}}^{p-1}+C_{2}\|u-v\|_{S, t_{0}}
$$

where $C_{1}$ and $C_{2}$ are constants depending only on $u_{0}, t_{0}, p, L$, and $\omega_{0}$. Therefore we obtain the continuity of $D$.

Finally, we will show that $D\left(B\left(t_{0}\right)\right)$ is relatively compact. By Lemma 1.11 it suffices to show that $D\left(B\left(t_{0}\right)\right)$ is uniformly bounded on $S \times\left[0, t_{0}\right]$ and equicontinuous on $\left[0, t_{0}\right]$. Since $D\left(B\left(t_{0}\right)\right) \in B\left(t_{0}\right)$, it is trivial that $D\left(B\left(t_{0}\right)\right)$ is uniformly bounded. On the other hand, it follows that for each $x \in S$,

$$
\left|D[u]\left(x, t_{1}\right)-D[u]\left(x, t_{2}\right)\right| \leq\left|t_{1}-t_{2}\right|\left[\omega_{0}\left(4\left\|u_{0}\right\|_{S, t_{0}}\right)^{p-1}+4 L\left\|u_{0}\right\|_{S, t_{0}}\right]
$$

for all $t_{1}, t_{2} \in\left[0, t_{0}\right]$ and $u \in B_{t_{0}}$, which implies that $D\left(B\left(t_{0}\right)\right)$ is equicontinuous on $\left[0, t_{0}\right]$. Hence $D\left(B\left(t_{0}\right)\right)$ is relatively compact by Lemma 1.11 . Therefore by the Schauder fixed point theorem there exists $u \in B\left(t_{0}\right)$ satisfying $D[u]=u$ and the boundary condition $B[u]=0$. It is clear that $u$ is the solution to equation (2). On the other hand, it is easy to see that $u$ is bounded. Moreover, $u(x, \cdot)$ is continuous on $\left[0, t_{0}\right]$ and differentiable in $\left(0, t_{0}\right)$ for each $x \in \bar{S}$ by the definition of $D$ and the boundary condition $B[u]=0$.

Now we state two types of comparison principles.

Theorem 1.13 (Comparison principle) Let $T>0(T$ may be $+\infty)$ and $p>1$, and let $f$ be locally Lipschitz continuous on $\mathbb{R}$. Suppose that real-valued functions $u(x, \cdot), v(x, \cdot) \in$ $C[0, T)$ are differentiable in $(0, T)$ for each $x \in \bar{S}$ and satisfy

$$
\begin{cases}u_{t}(x, t)-\Delta_{p, \omega} u(x, t)-f(u(x, t)) & \\ \quad \geq v_{t}(x, t)-\Delta_{p, \omega} v(x, t)-f(v(x, t)), & (x, t) \in S \times(0, T), \\ B[u] \geq B[v] & \text { on } \partial S \times[0, T), \\ u(x, 0) \geq v(x, 0), & x \in \bar{S} .\end{cases}
$$

Then $u(x, t) \geq v(x, t)$ for all $(x, t) \in \bar{S} \times[0, T)$.

Proof Let $T^{\prime}>0$ be arbitrarily given with $T^{\prime}<T$. Since $f$ is locally Lipschitz continuous on $\mathbb{R}$, there exists $L>0$ such that

$$
|f(a)-f(b)| \leq L|a-b|, \quad a, b \in[-m, m],
$$


where $m=\max _{x \in \bar{S}} \max _{0 \leq t \leq T^{\prime}}\{|u(x, t)|,|v(x, t)|\}$. Let $\tilde{u}, \tilde{v}: \bar{S} \times\left[0, T^{\prime}\right] \rightarrow \mathbb{R}$ be the functions defined by

$$
\begin{array}{ll}
\tilde{u}(x, t):=e^{-2 L t} u(x, t), & (x, t) \in \bar{S} \times\left[0, T^{\prime}\right] . \\
\tilde{v}(x, t):=e^{-2 L t} v(x, t), & (x, t) \in \bar{S} \times\left[0, T^{\prime}\right] .
\end{array}
$$

Then from (4) we have

$$
\begin{aligned}
& {\left[\tilde{u}_{t}(x, t)-\tilde{v}_{t}(x, t)\right]-e^{2 L(p-2) t}\left[\Delta_{p, \omega} \tilde{u}(x, t)-\Delta_{p, \omega} \tilde{v}(x, t)\right]} \\
& \quad+2 L[\tilde{u}(x, t)-\tilde{v}(x, t)]-e^{-2 L t}[f(u(x, t))-f(v(x, t))] \geq 0
\end{aligned}
$$

for all $(x, t) \in S \times\left(0, T^{\prime}\right]$.

We recall that $\tilde{u}(x, \cdot)$ and $\tilde{v}(x, \cdot)$ are continuous on $\left[0, T^{\prime}\right]$ for each $x \in \bar{S}$ and $\bar{S}$ is finite. Hence we can find $\left(x_{0}, t_{0}\right) \in \bar{S} \times\left[0, T^{\prime}\right]$ such that

$$
(\tilde{u}-\tilde{v})\left(x_{0}, t_{0}\right)=\min _{x \in \bar{S}} \min _{0 \leq t \leq T^{\prime}}(\tilde{u}-\tilde{v})(x, t),
$$

which implies that

$$
\tilde{v}\left(y, t_{0}\right)-\tilde{v}\left(x_{0}, t_{0}\right) \leq \tilde{u}\left(y, t_{0}\right)-\tilde{u}\left(x_{0}, t_{0}\right), \quad y \in \bar{S} .
$$

Then now we have only to show that $(\tilde{u}-\tilde{v})\left(x_{0}, t_{0}\right) \geq 0$.

Suppose that, on the contrary, $(\tilde{u}-\tilde{v})\left(x_{0}, t_{0}\right)<0$. Assume that $x_{0} \in \partial S$. Then we see that

$$
\begin{aligned}
0 \leq & \mu\left(x_{0}\right) \sum_{x \in S}\left[\left|\tilde{u}\left(x_{0}, t_{0}\right)-\tilde{u}\left(x, t_{0}\right)\right|^{p-2}\left(\tilde{u}\left(x_{0}, t_{0}\right)-\tilde{u}\left(x, t_{0}\right)\right)\right. \\
& \left.-\left|\tilde{v}\left(x_{0}, t_{0}\right)-\tilde{v}\left(x, t_{0}\right)\right|^{p-2}\left(\tilde{v}\left(x_{0}, t_{0}\right)-\tilde{v}\left(x, t_{0}\right)\right)\right] \omega\left(x_{0}, x\right) \\
& +\sigma\left(x_{0}\right)\left(\tilde{u}\left(x_{0}, t_{0}\right)-\tilde{v}\left(x_{0}, t_{0}\right)\right) .
\end{aligned}
$$

Therefore, if $\sigma\left(x_{0}\right)>0$, then equation (8) is negative, which leads to a contradiction. If $\sigma\left(x_{0}\right)=0$, then we have

$$
\tilde{u}\left(x_{0}, t_{0}\right)-\tilde{v}\left(x_{0}, t_{0}\right)=\tilde{u}\left(x, t_{0}\right)-\tilde{v}\left(x, t_{0}\right)
$$

for all $x \in S$. Hence there exists $x_{1} \in S$ such that

$$
\tilde{u}\left(x_{0}, t_{0}\right)-\tilde{v}\left(x_{0}, t_{0}\right)=\tilde{u}\left(x_{1}, t_{0}\right)-\tilde{v}\left(x_{1}, t_{0}\right) .
$$

Hence we may choose $x_{0} \in S$. Moreover, since $\tilde{u}(x, 0)-\tilde{v}(x, 0) \geq 0$ on $\bar{S}$, we have $\left(x_{0}, t_{0}\right) \in$ $S \times\left(0, T^{\prime}\right]$. Then we obtain from (7) that

$$
\Delta_{p, \omega} \tilde{u}\left(x_{0}, t_{0}\right)-\Delta_{p, \omega} \tilde{v}\left(x_{0}, t_{0}\right) \geq 0
$$

and from the differentiability of $(\tilde{u}-\tilde{v})(x, t)$ in $\left(0, T^{\prime}\right]$ for each $x \in \bar{S}$ it follows that

$$
\left(\tilde{u}_{t}-\tilde{v}_{t}\right)\left(x_{0}, t_{0}\right) \leq 0
$$


According to (5), we have

$$
\begin{aligned}
2 L & {\left[\tilde{u}\left(x_{0}, t_{0}\right)-\tilde{v}\left(x_{0}, t_{0}\right)\right]-e^{-2 L t_{0}}\left[f\left(u\left(x_{0}, t_{0}\right)\right)-f\left(v\left(x_{0}, t_{0}\right)\right)\right] } \\
& \leq 2 L\left[\tilde{u}\left(x_{0}, t_{0}\right)-\tilde{v}\left(x_{0}, t_{0}\right)\right]+L e^{-2 L t_{0}}\left|u\left(x_{0}, t_{0}\right)-v\left(x_{0}, t_{0}\right)\right| \\
& =2 L\left[\tilde{u}\left(x_{0}, t_{0}\right)-\tilde{v}\left(x_{0}, t_{0}\right)\right]+L\left|\tilde{u}\left(x_{0}, t_{0}\right)-\tilde{v}\left(x_{0}, t_{0}\right)\right| \\
& =L\left[\tilde{u}\left(x_{0}, t_{0}\right)-\tilde{v}\left(x_{0}, t_{0}\right)\right]<0,
\end{aligned}
$$

since $\tilde{u}\left(x_{0}, t_{0}\right)<\tilde{v}\left(x_{0}, t_{0}\right)$. Combining (9), (10), and (11), we obtain that

$$
\begin{aligned}
& \tilde{u}\left(x_{0}, t_{0}\right)-\tilde{v}\left(x_{0}, t_{0}\right)-\left[\Delta_{p, \omega} \tilde{u}\left(x_{0}, t_{0}\right)-\Delta_{p, \omega} \tilde{v}\left(x_{0}, t_{0}\right)\right] \\
& \quad+2 L\left[\tilde{u}\left(x_{0}, t_{0}\right)-\tilde{v}\left(x_{0}, t_{0}\right)\right]-e^{-2 L t_{0}}\left[f\left(u\left(x_{0}, t_{0}\right)\right)-f\left(v\left(x_{0}, t_{0}\right)\right)\right]<0,
\end{aligned}
$$

which contradicts (6). Therefore $\tilde{u}(x, t) \geq \tilde{v}(x, t)$ for all $(x, t) \in S \times\left(0, T^{\prime}\right]$, so that we get $u(x, t) \geq v(x, t)$ for all $(x, t) \in \bar{S} \times[0, T)$, since $T^{\prime}<T$ is arbitrary.

When $p \geq 2$, we obtain a strong comparison principle.

Theorem 1.14 (Strong comparison principle) Let $T>0(T$ maybe $+\infty)$ and $p \geq 2$, and let $f$ be locally Lipschitz continuous on $\mathbb{R}$. Suppose that real-valued functions $u(x, \cdot), v(x, \cdot) \in$ $C[0, T)$ are differentiable in $(0, T)$ for each $x \in \bar{S}$ and satisfy

$$
\begin{cases}u_{t}(x, t)-\Delta_{p, \omega} u(x, t)-f(u(x, t)) & \\ \quad \geq v_{t}(x, t)-\Delta_{p, \omega} v(x, t)-f(v(x, t)), & (x, t) \in S \times(0, T), \\ B[u] \geq B[v] & \text { on } \partial S \times[0, T), \\ u(x, 0) \geq v(x, 0), & x \in \bar{S} .\end{cases}
$$

If $u\left(x^{*}, 0\right)>v\left(x^{*}, 0\right)$ for some $x^{*} \in S$, then $u(x, t)>v(x, t)$ for all $(x, t) \in S \cup \Gamma \times(0, T)$.

Proof First, note that $u \geq v$ on $\bar{S} \times[0, T)$ by the previous theorem. Let $T^{\prime}>0$ be arbitrarily given with $T^{\prime}<T$. Since $f$ is locally Lipschitz continuous on $\mathbb{R}$, there exists $L>0$ such that

$$
|f(a)-f(b)| \leq L|a-b|, \quad a, b \in[-m, m]
$$

where $m=\max _{x \in \bar{S}} \max _{0 \leq t \leq T^{\prime}}\{|u(x, t)|,|v(x, t)|\}$. Let $\tau: \bar{S} \times\left[0, T^{\prime}\right] \rightarrow \mathbb{R}$ be the function defined by

$$
\tau(x, t):=u(x, t)-v(x, t), \quad(x, t) \in \bar{S} \times\left[0, T^{\prime}\right] .
$$

Then $\tau(x, t) \geq 0$ for all $(x, t) \in \bar{S} \times\left[0, T^{\prime}\right]$. From inequality (12) we have

$$
\tau_{t}\left(x^{*}, t\right)-\Delta_{p, \omega} u\left(x^{*}, t\right)-\Delta_{p, \omega} v\left(x^{*}, t\right)-\left[f\left(u\left(x^{*}, t\right)\right)-f\left(v\left(x^{*}, t\right)\right)\right] \geq 0
$$


for all $0<t \leq T^{\prime}$. Then by the mean value theorem, for each $y \in \bar{S}$ and $0 \leq t \leq T^{\prime}$, it follows that

$$
\begin{aligned}
& \left|u(y, t)-u\left(x^{*}, t\right)\right|^{p-2}\left[u(y, t)-u\left(x^{*}, t\right)\right]-\left|v(y, t)-v\left(x^{*}, t\right)\right|^{p-2}\left[v(y, t)-v\left(x^{*}, t\right)\right] \\
& \quad=(p-1)\left|\zeta\left(x^{*}, y, t\right)\right|^{p-2}[\tau(y, t)-\tau(x, t)]
\end{aligned}
$$

where $\left|\zeta\left(x^{*}, y, t\right)\right| \leq 2 \max _{x \in \bar{S}} \max _{0 \leq t \leq T^{\prime}}|u(x, t)|,|v(x, t)|$. By (13) and (15) inequality (14) becomes

$$
\begin{aligned}
& \tau_{t}\left(x^{*}, t\right) \\
& \quad \geq-d_{\omega} x^{*}(p-1)[2 M]^{p-2} \tau\left(x^{*}, t\right)-L\left|\tau\left(x^{*}, t\right)\right| \\
& \quad=-\left(d_{\omega} x^{*}(p-1)[2 M]^{p-2}+L\right) \tau\left(x^{*}, t\right) .
\end{aligned}
$$

This implies

$$
\tau\left(x^{*}, t\right) \geq \tau\left(x^{*}, 0\right) e^{-\left(d_{\omega} x^{*}(p-1)[2 M]^{p-2}+L\right) t}>0, \quad t \in\left(0, T^{\prime}\right],
$$

since $\tau\left(x^{*}, 0\right)>0$. Now suppose there exists $\left(x_{0}, t_{0}\right) \in S \cup \Gamma \times\left(0, T^{\prime}\right]$ such that

$$
\tau\left(x_{0}, t_{0}\right)=\min _{x \in S \cup \Gamma, 0<t \leq T^{\prime}} \tau(x, t)=0 .
$$

Case 1: $x_{0} \in S$.

Since $\tau\left(x_{0}, t_{0}\right) \leq \tau(x, t)$ for all $(x, t) \in \bar{S} \times\left[0, T^{\prime}\right]$, we have

$$
\tau_{t}\left(x_{0}, t_{0}\right) \leq 0
$$

and

$$
\Delta_{p, \omega} u\left(x_{0}, t_{0}\right)-\Delta_{p, \omega} v\left(x_{0}, t_{0}\right) \geq 0
$$

Hence from inequality (12) we obtain

$$
0 \leq \tau_{t}\left(x_{0}, t_{0}\right)-\Delta_{p, \omega} u\left(x_{0}, t_{0}\right)+\Delta_{p, \omega} v\left(x_{0}, t_{0}\right) \leq 0
$$

Therefore we have

$$
\Delta_{p, \omega} u\left(x_{0}, t_{0}\right)-\Delta_{p, \omega} v\left(x_{0}, t_{0}\right)=0,
$$

which implies that $\tau\left(y, t_{0}\right)=0$ for all $y \in \bar{S}$ with $y \sim x_{0}$. Now, for any $x \in \bar{S}$, there exists a path

$$
x_{0} \sim x_{1} \sim \ldots \sim x_{n} \sim x
$$

since $\bar{S}$ is connected. By applying the same argument as before inductively we see that $\tau\left(x, t_{0}\right)=0$ for every $x \in \bar{S}$, which is a contradiction to (16). 
Case 2: $x_{0} \in \Gamma$.

By the boundary condition in (12) we have

$$
\begin{aligned}
& \mu(x)\left[\frac{\partial u}{\partial_{p} n}\left(x_{0}, t_{0}\right)-\frac{\partial v}{\partial_{p} n}\left(x_{0}, t_{0}\right)\right] \\
& \quad \geq \sigma(x)\left[\left|u\left(x_{0}, t_{0}\right)\right|^{p-2} u\left(x_{0}, t_{0}\right)-|v(x, t)|^{p-2} v\left(x_{0}, t_{0}\right)\right]=0
\end{aligned}
$$

from which it follows that

$$
u\left(x_{0}, t_{0}\right)-u\left(x_{1}, t_{0}\right) \geq v\left(x_{0}, t_{0}\right)-v\left(x_{1}, t_{0}\right)
$$

for some $x_{1} \in S$ with $x_{0} \sim x_{1}$. This means that $\tau\left(x_{1}, t_{0}\right)=0$, which contradicts to Case 1 . Hence we finally obtain that $u(x, t)>v(x, t)$ for all $(x, t) \in S \times(0, T)$, since $T^{\prime}<T$ is arbitrary.

Note that by the comparison principle, if $f(0)=0$, then solutions $u$ to equation (2) are nonnegative. On the other hand, it is natural that $f$ is assumed to be positive on $(0,+\infty)$ when we deal with the blow-up theory. Hence we always assume that $f$ is a locally Lipschitz continuous function on $\mathbb{R}$, which is positive in $(0,+\infty)$ and $f(0)=0$. Moreover, we assume that the initial data $u_{0}$ is nontrivial and nonnegative.

\section{Blow-up: the concavity method}

In this section, we discuss the blow-up phenomena of the solutions to equation (2), which is the main part of this paper.

Definition 2.1 (Blow-up) We say that a solution $u$ to equation (2) blows up at finite time $T>0$ if there exists $x \in S$ such that $|u(x, t)| \rightarrow+\infty$ as $t \nearrow T^{-}$or, equivalently, $\sum_{x \in S}|u(x, t)| \rightarrow+\infty$ as $t \nearrow T^{-}$.

To state and prove our result, we introduce the following condition:

$$
\left(C_{p}\right) \quad \alpha F(u) \leq u f(u)+\beta u^{p}+\gamma, \quad u>0,
$$

for some $\alpha>2, \beta \geq 0$, and $\gamma>0$ with $0 \leq \beta \leq \frac{(\alpha-p) \lambda_{p, 0}}{p}$.

Remark 2.2 We have the fact that $\lambda_{p, 0}=0$ if and only if $\sigma \equiv 0$ (see [12]). Therefore we can easily obtain that the condition on $\alpha$ in $\left(C_{p}\right)$ depends on the boundary condition and $p>1$ as follows:

(i) If $\sigma \equiv 0$, then $\alpha>2$ for all $p>1$.

(ii) If $\sigma \not \equiv 0$, then $\alpha>2$ for all $1<p \leq 2$.

(iii) If $\sigma \neq \equiv 0$, then $\alpha \geq p$ for all $p>2$.

We now state the main theorem of this paper: 
Theorem 2.3 For $p>1$ and the function $f$ with hypothesis $\left(C_{p}\right)$, if the initial data $u_{0}$ satisfies

$$
\begin{aligned}
& -\frac{1}{2 p} \sum_{x, y \in \bar{S}}\left|u_{0}(x)-u_{0}(y)\right|^{p} \omega(x, y)-\frac{1}{p} \sum_{z \in \Gamma} \frac{\sigma(z)}{\mu(z)}\left|u_{0}(z)\right|^{p} \\
& \quad+\sum_{x \in S}\left[F\left(u_{0}(x)\right)-\gamma\right]>0
\end{aligned}
$$

then the solutions $u$ to equation (2) blow up at finite time $T^{*}$ in the sense that

$$
\lim _{t \rightarrow T^{*}} \sum_{x \in S} u^{2}(x, t)=+\infty
$$

where $\gamma$ is the constant in e condition $\left(C_{p}\right)$.

Proof First, let us define functionals by

$$
A(t):=\sum_{x \in S} u^{2}(x, t), \quad t \geq 0
$$

and

$$
\begin{aligned}
B(t):= & -\frac{1}{2 p} \sum_{x, y \in \bar{S}}|u(x, t)-u(y, t)|^{p} \omega(x, y)-\frac{1}{p} \sum_{z \in \Gamma} \frac{\sigma(z)}{\mu(z)}|u(z, t)|^{p} \\
& +\sum_{x \in S}[F(u(x, t))-\gamma], \quad t \geq 0 .
\end{aligned}
$$

Then we have from equation (2) and Lemma 1.7 that

$$
\begin{aligned}
A^{\prime}(t)= & 2 \sum_{x \in S} u(x, t)\left[\Delta_{p, \omega} u(x, t)+f(u(x, t))\right] \\
= & 2 \sum_{x \in \bar{S}} u(x, t) \Delta_{p, \omega} u(x, t)+2 \sum_{z \in \Gamma} u(z, t) \frac{\partial u}{\partial_{p} \eta}(z, t)+2 \sum_{x \in S} u(x, t) f(u(x, t)) \\
= & -\sum_{x, y \in \bar{S}}|u(x, t)-u(y, t)|^{p} \omega(x, y)-2 \sum_{z \in \Gamma} \frac{\sigma(z)}{\mu(z)}|u(z, t)|^{p} \\
& +2 \sum_{x \in \bar{S}} u(x, t) f(u(x, t)) .
\end{aligned}
$$

Applying condition $\left(C_{p}\right)$ and Lemma 1.8, we can see that (18) implies

$$
\begin{aligned}
A^{\prime}(t) \geq & 2 \sum_{x \in S}\left[\alpha F(u(x, t))-\beta u^{p}(x, t)-\gamma\right]-\sum_{x, y \in \bar{S}}|u(x, t)-u(y, t)|^{p} \omega(x, y) \\
& -2 \sum_{z \in \Gamma} \frac{\sigma(z)}{\mu(z)}|u(z, t)|^{p} \\
\geq & 2 \alpha B(t)-2 \beta \sum_{x \in S} u^{p}(x, t)+\left(\frac{\alpha}{p}-1\right) \sum_{x, y \in \bar{S}}|u(x, t)-u(y, t)|^{p} \omega(x, y)
\end{aligned}
$$




$$
\begin{aligned}
& +2\left(\frac{\alpha}{p}-1\right) \sum_{z \in \Gamma} \frac{\sigma(z)}{\mu(z)}|u(z, t)|^{p} \\
\geq & 2 \alpha B(t)+2\left[\frac{(\alpha-p) \lambda_{p, 0}}{p}-\beta\right] \sum_{x \in S} u^{p}(x, t) \\
\geq & 2 \alpha B(t) .
\end{aligned}
$$

Here it is easy to see that if $\lambda_{p, 0}=0$ or $\alpha=p$, then $\beta=0$. Therefore, even though $\lambda_{p, 0}=0$ or $\alpha=p$, (19) is true.

On the other hand, we have from equation (2) and Lemma 1.7 that

$$
\begin{aligned}
B^{\prime}(t)= & -\frac{1}{2} \sum_{x, y \in \bar{S}}|u(y, t)-u(x, t)|^{p-2}[u(y, t)-u(x, t)]\left[u_{t}(y, t)-u_{t}(x, t)\right] \omega(x, y) \\
& -\sum_{z \in \Gamma} \frac{\sigma(z)}{\mu(z)}|u(z, t)|^{p-2} u(z, t) u_{t}(z, t)+\sum_{x \in S} f(u(x, t)) u_{t}(x, t) \\
= & \sum_{x \in \bar{S}} \Delta_{p, \omega} u(x, t) u_{t}(x, t)+\sum_{z \in \partial S} \frac{\partial u}{\partial_{p} n}(z, t) u_{t}(z, t)+\sum_{x \in S} f(u(x, t)) u_{t}(x, t) \\
= & \sum_{x \in S} u_{t}(x, t)\left[\Delta_{p, \omega} u(x, t)+f(u(x, t))\right] \\
= & \sum_{x \in S} u_{t}^{2}(x, t) \geq 0 .
\end{aligned}
$$

Now we will show that

$$
\frac{d}{d t}\left[A^{-\frac{\alpha}{2}}(t) B(t)\right]=-\frac{\alpha}{2} A^{-\frac{\alpha}{2}-1} A^{\prime}(t) B(t)+A^{-\frac{\alpha}{2}} B^{\prime}(t) \geq 0
$$

for all $t>0$. Using the Schwarz inequality, from (19) and (20) we obtain that

$$
\begin{aligned}
\frac{\alpha}{2} A^{\prime}(t) B(t) & \leq \frac{1}{4}\left[A^{\prime}(t)\right]^{2}=\left[\sum_{x \in S} u(x, t) u_{t}(x, t)\right]^{2} \leq \sum_{x \in S} u^{2}(x, t) \sum_{x \in S} u_{t}^{2}(x, t) \\
& =A(t) B^{\prime}(t)
\end{aligned}
$$

for all $t>0$. Therefore inequality (21) is true, which implies that

$$
\frac{1}{2 \alpha} A^{-\frac{\alpha}{2}}(t) A^{\prime}(t) \geq A^{-\frac{\alpha}{2}}(t) B(t) \geq A^{-\frac{\alpha}{2}}(0) B(0)>0 .
$$

Solving the differential inequality (22), we obtain

$$
A(t) \geq\left[\frac{1}{-(\alpha-2) \alpha A^{-\frac{\alpha}{2}}(0) B(0) t+A^{\frac{2-\alpha}{2}}(0)}\right]^{\frac{2}{\alpha-2}} .
$$

Hence $A(t)$ blows up in finite time $T$ with $0<T \leq \frac{A(0)}{(\alpha-2) \alpha B(0)}$. 
Remark 2.4 The blow-up time can be estimated roughly as

$$
0<T \leq \frac{\frac{1}{(\alpha-2) \alpha} \sum_{x \in S} u_{0}^{2}(x)}{-\frac{\sum_{x, y \in \bar{S}}\left|u_{0}(x)-u_{0}(y)\right|^{p} \omega(x, y)}{2 p}-\frac{\sum_{z \in \Gamma} \frac{\sigma(z)}{\mu(z)} u_{0}^{p}(z)}{p}+\sum_{x \in S}\left[F\left(u_{0}(x)\right)-\gamma\right]} .
$$

Remark 2.5 Chung and Choi [11] obtained the blow-up results for equation (2) under the Dirichlet boundary condition in the continuous setting, where $p \geq 2$ by using condition $\left(C_{p}\right)$. In fact, their condition had the assumption $\alpha>p$, which is one of the main differences to us.

\section{Discussion on condition $\left(C_{p}\right)$ with the initial data conditions}

In this section, we compare conditions $\left(A_{p}\right),\left(B_{p}\right)$, and $\left(C_{p}\right)$ and discuss the role of $B(0)>0$.

First, we consider the Neumann boundary condition $\sigma \equiv 0$. Summing up over $S$ to equation (2), we have

$$
\begin{aligned}
\sum_{x \in S} u_{t}(x, t) & =\sum_{x \in \bar{S}} \Delta_{p, \omega} u(x, t)-\sum_{z \in \partial S} \Delta_{p, \omega} u(z, t)+\sum_{x \in S} f(u(x, t)) \\
& =\sum_{x \in S} f(u(x, t)) .
\end{aligned}
$$

From this equality we can obtain that the time behavior of $\sum_{x \in S} u(x, t)$ is determined by $\sum_{x \in S} f(u(x, t))$. Therefore by the definition of the blow-up we can expect that the blow-up condition for the solution $u$ depends only on $f$, not on $p$. On the other hand, for all $p>1$, condition $\left(C_{p}\right)$ is represented by

$$
(2+\epsilon) F(u) \leq u f(u)+\gamma
$$

for some $\epsilon>0$ and $\gamma>0$, which also does not depend on $p$.

From now on we consider the boundary condition $\sigma \not \equiv 0$. Let us recall the following conditions:

for $1<p \leq 2$,

$$
\begin{array}{ll}
\left(A_{p}\right) & (2+\epsilon) F(u) \leq u f(u), \\
\left(B_{p}\right) & (2+\epsilon) F(u) \leq u f(u)+\gamma, \\
\left(C_{p}\right) & (2+\epsilon) F(u) \leq u f(u)+\beta u^{p}+\gamma,
\end{array}
$$

where

$$
\epsilon>0, \quad 0 \leq \beta \leq \frac{(2+\epsilon-p) \lambda_{p, 0}}{p}, \quad \text { and } \quad \gamma>0,
$$

and for $p>2$,

$$
\begin{aligned}
& \left(A_{p}\right) \quad(p+\epsilon) F(u) \leq u f(u), \\
& \left(B_{p}\right) \quad(p+\epsilon) F(u) \leq u f(u)+\gamma,
\end{aligned}
$$




$$
\left(C_{p}\right) \quad(p+\epsilon) F(u) \leq u f(u)+\beta u^{p}+\gamma,
$$

where

$$
\epsilon \geq 0, \quad 0 \leq \beta \leq \frac{\epsilon \lambda_{p, 0}}{p}, \quad \text { and } \quad \gamma>0
$$

for every $u \geq 0$. Here $F(u):=\int_{0}^{u} f(s) d s$.

It is easy to see that $\left(A_{p}\right)$ implies $\left(B_{p}\right)$ and in turn $\left(B_{p}\right)$ implies $\left(C_{p}\right)$. In fact, the first eigenvalue $\lambda_{p, 0}$, which depends on the domain, is not contained in conditions $\left(A_{p}\right)$ and $\left(B_{p}\right)$. However, condition $\left(C_{p}\right)$ depends on the domain due to the term $\beta u^{p}$. From this point of view, condition $\left(C_{p}\right)$ can be understood as a refinement of $\left(B_{p}\right)$, corresponding to the domain. On the contrary, if a function $f$ satisfies $\left(C_{p}\right)$ for every domain, then the first eigenvalue $\lambda_{p, 0}$ can be arbitrarily small so that condition $\left(C_{p}\right)$ gets arbitrarily closer to $\left(B_{p}\right)$.

Remark 3.1 In fact, there have been efforts to obtain the condition $\epsilon=0$ in the continuous analogue. For example, Junning studied the blow-up solutions to equation (2) in the continuous setting under the Dirichlet boundary condition with the assumption $\epsilon=0$ in $\left(A_{p}\right)$ and the initial data $u_{0}$ satisfying

$$
-\frac{1}{p} \int_{\Omega}\left|\nabla u_{0}(x)\right|^{p} d x+\int_{\Omega} F\left(u_{0}(x)\right) d x \geq \frac{4(p-1)}{T(p-2)^{2} p} \int_{\Omega} u_{0}^{2}(x) d x
$$

where $p>2$ and $\Omega \subset \mathbb{R}^{N}$ (see [7]). From this point of view, for $p>2$, our condition $\epsilon \geq 0$ with $B(0)>0$ improves the conventional results.

Now we consider the cases $p>2$ and $1<p \leq 2$ to investigate conditions $\left(A_{p}\right),\left(B_{p}\right)$, and $\left(C_{p}\right)$.

Case 1: $p>2$.

Assuming that $\epsilon>0$, we obtain that condition $\left(C_{p}\right)$ is equivalent to

$$
\frac{d}{d u}\left(\frac{F(u)}{u^{p+\epsilon}}-\frac{\gamma}{p+\epsilon} \cdot \frac{1}{u^{p+\epsilon}}-\frac{\beta}{\epsilon} \cdot \frac{1}{u^{\epsilon}}\right) \geq 0, \quad u>0 .
$$

In a similar way, assuming that $\epsilon=0$, we have

$$
\frac{d}{d u}\left(\frac{F(u)}{u^{p}}-\frac{\gamma}{p} \cdot \frac{1}{u^{p}}\right) \geq 0, \quad u>0 .
$$

Hence (23) and (24) imply that for all $u>0$ and $p>2$,

$$
\begin{aligned}
& \left(A_{p}\right) \text { holds if and only if } F(u)=u^{p+\epsilon} h_{1}(u), \\
& \left(B_{p}\right) \text { holds if and only if } F(u)=u^{p+\epsilon} h_{2}(u)+b, \\
& \left(C_{p}\right) \text { holds if and only if } F(u)=u^{p+\epsilon} h_{3}(u)+a u^{p}+b,
\end{aligned}
$$

for some constants $\epsilon>0, a \geq 0$, and $b>0$ with $0 \leq a \leq \frac{\lambda_{p, 0}}{p}$, where $h_{1}, h_{2}$, and $h_{3}$ are nondecreasing function on $(0,+\infty)$.

Case 2: $1<p \leq 2$. 
We obtain that $\left(C_{p}\right)$ is equivalent to

$$
\frac{d}{d u}\left(\frac{F(u)}{u^{2+\epsilon}}-\frac{\gamma}{2+\epsilon} \cdot \frac{1}{u^{2+\epsilon}}-\frac{\beta}{2+\epsilon-p} \cdot \frac{1}{u^{2+\epsilon-p}}\right) \geq 0, \quad u>0
$$

which implies that for all $u>0$ and $1<p \leq 2$,

$\left(A_{p}\right)$ holds if and only if $F(u)=u^{2+\epsilon} h_{1}(u)$,

$\left(B_{p}\right)$ holds if and only if $F(u)=u^{2+\epsilon} h_{2}(u)+b$,

$\left(C_{p}\right)$ holds if and only if $F(u)=u^{2+\epsilon} h_{3}(u)+a u^{p}+b$,

for some constants $\epsilon>0, a \geq 0$, and $b>0$ with $0 \leq a \leq \frac{\lambda_{p, 0}}{p}$, where $h_{1}, h_{2}$, and $h_{3}$ are nondecreasing function on $(0,+\infty)$.

In case 1 and case 2 , the constants $\epsilon, a$, and $b$ may be different in each case. Also, the nondecreasing function $h_{1}$ is nonnegative on $(0,+\infty)$, but $h_{2}$ and $h_{3}$ may be not nonnegative in general.

Theorem 3.2 For $p>1$, let $f$ be a real-valued function satisfying condition $\left(C_{p}\right)$. Suppose that $f(u) \geq \lambda u^{p-1}, u>0$, for some $\lambda>\lambda_{p, 0}$. Then the following statements are true.

(i) There exists $m>0$ such that $h_{3}(u)>0$ for $u \geq m$.

(ii) There exists $\zeta>0$ such that $f(u) \geq \zeta u^{\max \{p-1,1\}+\epsilon}, u \geq m$.

(iii) Conditions $\left(B_{p}\right)$ and $\left(C_{p}\right)$ are equivalent when $p \geq 2$.

Proof (i): First, it follows from the fact $F(u) \geq \frac{\lambda}{p} u^{p}>\frac{\lambda_{p, 0}}{p} u^{p}$ that

$$
u^{\max \{p, 2\}+\epsilon} h_{3}(u)=F(u)-a u^{p}-b \geq \frac{\lambda-\lambda_{p, 0}}{p} u^{p}-b,
$$

which goes to $+\infty$ as $u \rightarrow+\infty$. Therefore we can find $m>0$ such that $h_{3}(m)>0$.

(ii): (i) implies that

$$
F(u) \geq u^{\max \{p, 2\}+\epsilon} h_{3}(u), \quad u \geq m .
$$

Putting it into condition $\left(C_{p}\right)$, we obtain

$$
\alpha u^{\max \{p, 2\}+\epsilon} h_{3}(m) \leq u f(u)+\beta u^{p}+\gamma .
$$

Hence we obtain that

$$
\alpha u^{\max \{p-1,1\}+\epsilon} h_{3}(m) \leq f(u)+\beta u^{p-1}+\frac{\gamma}{u} \leq\left(1+\frac{\beta}{\lambda_{p, 0}}\right) f(u)+\gamma, \quad u \geq m>0,
$$

which gives

$$
f(u) \geq \zeta u^{1+\epsilon}, \quad u \geq m>0,
$$

for some $\zeta>0$. 
(iii): Now consider the case $p \geq 2$. Then it is trivial that $\left(B_{p}\right)$ and $\left(C_{p}\right)$ are equivalent when $\epsilon=0$. Therefore we may assume that $\epsilon>0$. Since $0 \leq \beta \leq \frac{\epsilon \lambda_{p, 0}}{p}$ and $f(u) \geq \lambda u>\lambda_{p, 0} u, u>0$, it follows from $\left(C_{p}\right)$ that

$$
\epsilon_{1} F(u)+\left(p+\epsilon_{2}\right) F(u) \leq u f(u)+\frac{\epsilon \lambda_{p, 0}}{p} u^{p}+\gamma
$$

where $\epsilon_{1}=\frac{\epsilon \lambda_{p, 0}}{\lambda}>0$ and $\epsilon_{2}=\epsilon-\epsilon_{1}>0$. This implies that for every $u>0$,

$$
\begin{aligned}
u f(u)+\gamma & \geq\left(p+\epsilon_{2}\right) F(u)+\epsilon_{1} \int_{0}^{u}\left[f(s)-\lambda s^{p-1}\right] d s \\
& \geq\left(p+\epsilon_{2}\right) F(u)
\end{aligned}
$$

which implies $\left(B_{p}\right)$.

In general, only condition $\left(C_{p}\right)$ may not guarantee the blow-up solutions for every initial data $u_{0}$. Therefore, from now on, we are going to discuss when we can find initial data $u_{0}$ that satisfies $B(0)>0$.

Lemma 3.3 Let $p>1$. If there exists $v_{0}>0$ such that $F\left(v_{0}\right)>\frac{\omega_{0}}{p} v_{0}^{p}+\gamma_{1}$, where $\gamma_{1} \geq \gamma$, then there exists the initial data $u_{0}$ such that $B(0)>0$. Here $\omega_{0}:=\max _{x \in S} d_{\omega} x$.

Proof First of all, there exist $a, b>0$ with $0<a<b$ such that $F(v)>\frac{\omega_{0}}{p} v^{p}+\gamma_{1}, v \in(a, b)$, since $F$ is continuous on $[0,+\infty)$. Now, we consider the function $u_{0}(x)$ satisfying

$$
\begin{cases}a<u_{0}(x)<b, & x \in S, \\ 0<u_{0}(x)<b, & x \in \Gamma, \\ u_{0}(x)=0, & x \in \partial S \backslash \Gamma,\end{cases}
$$

which satisfies the boundary condition $B\left[u_{0}\right]=0$. Then we obtain that

$$
\begin{aligned}
B(0)= & \frac{1}{p} \sum_{x \in S} \sum_{y \in \bar{S}}\left|u_{0}(y)-u_{0}(x)\right|^{p-2}\left[u_{0}(y)-u_{0}(x)\right] u_{0}(x) \omega(x, y) \\
& +\sum_{x \in S}\left[F\left(u_{0}(x)\right)-\gamma\right] \\
\geq & -\frac{1}{p} \sum_{x \in S} \sum_{y \in \bar{S}} u_{0}^{p}(x) \omega(x, y)+\sum_{x \in S}\left[F\left(u_{0}(x)\right)-\gamma\right] \\
= & -\frac{1}{p} \sum_{x \in S} u_{0}^{p}(x) d_{\omega} x+\sum_{x \in S}\left[F\left(u_{0}(x)\right)-\gamma\right] \\
\geq & \sum_{x \in S}\left[F\left(u_{0}(x)\right)-\frac{\omega_{0}}{p} u_{0}^{p}(x)\right]-\gamma|S| \\
> & \gamma_{1}|S|-\gamma|S| \geq 0,
\end{aligned}
$$

where $|S|$ denotes the number of vertices in $S$. 
Corollary 3.4 The following statements are true.

(i) If there exists $(a, b)$ such that $F(v)>\frac{\omega_{0}}{p} v^{p}+\gamma_{1}, \gamma_{1} \geq \gamma$ for every $v \in(a, b)$, then for every $u_{0}$ satisfying the boundary condition $B\left[u_{0}\right]=0$ such that

$$
\begin{cases}a<u_{0}(x)<b, & x \in S, \\ 0<u_{0}(x)<b, & x \in \Gamma, \\ u_{0}(x)=0, & x \in \partial S \backslash \Gamma,\end{cases}
$$

we see that $B(0)>0$.

(ii) If $F(v)>\omega_{0} v^{\max \left\{2+\epsilon_{1}, p\right\}}+\gamma_{1}, \epsilon_{1}>0, \gamma_{1} \geq \gamma$, for every $v \in(0,+\infty)$ on $S \cup \Gamma$, then the solutions blow up for every initial data $u_{0}>0$ on $S \cup \Gamma$. Here $\omega_{0}:=\max _{x \in S} d_{\omega} x$.

\section{Acknowledgements}

The authors would like to express thanks to anonymous reviewers for their excellent suggestions.

\section{Funding}

The first author was supported by Basic Science Research Program through the National Research Foundation of Korea (NRF) funded by the Ministry of Education (NRF-2015R1D1A1A01059561). The third author was supported by the Ministry of Education of the Republic of Korea and the National Research Foundation of Korea (BK21PLUS) (NRF-22A20130000051).

\section{Availability of data and materials}

Not applicable.

\section{Competing interests}

The authors declare that they have no competing interests.

\section{Authors' contributions}

The authors contributed and approved the final manuscript.

\section{Author details}

'National Institute for Mathematical Sciences, Daejeon, Republic of Korea. ${ }^{2}$ Department of Mathematics, Sogang University, Seoul, Republic of Korea. ${ }^{3}$ Present address: Department of Mathematical Sciences, Seoul National University, Seoul, Republic of Korea.

\section{Publisher's Note}

Springer Nature remains neutral with regard to jurisdictional claims in published maps and institutional affiliations.

Received: 15 May 2019 Accepted: 14 November 2019 Published online: 22 November 2019

\section{References}

1. Elmoataz, A., Lezoray, O., Bougleux, S.: Nonlocal discrete regularization on weighted graphs: a framework for image and manifold processing. IEEE Trans. Image Process. 17(7), 1047-1060 (2008)

2. He, Z: On the existence of positive solutions of $p$-Laplacian difference equations. J. Comput. Appl. Math. 161(1), 193-201 (2003)

3. Candito, P., Guivannelli, N.: Multiple solutions for a discrete boundary value problem involving the $p$-Laplacian. Comput. Math. Appl. 56(4), 959-964 (2008)

4. Levine, H.A.: Some nonexistence and instability theorems for formally parabolic equations of the form $P u_{t}=-A u+\mathcal{F}(u)$. Arch. Ration. Mech. Anal. 51, 371-386 (1973)

5. Philippin, G.A., Proytcheva, V: Some remarks on the asymptotic behaviour of the solutions of a class of parabolic problems. Math. Methods Appl. Sci. 29, 297-307 (2006)

6. Messaoudi, S.A.: A note on blow up of solutions of a quasilinear heat equation with vanishing initial energy. J. Math. Anal. Appl. 273, 243-247 (2002)

7. Junning, Z.: Existence and nonexistence of solutions for $u_{t}=\operatorname{div}\left(|\nabla u|^{p-2} \nabla u\right)+f(\nabla u, u, x, t)$. J. Math. Anal. Appl. 172, 130-146 (1993)

8. Ding, J., Hu, H.: Blow-up and global solutions for a class of nonlinear reaction diffusion equations under Dirichlet boundary conditions. J. Math. Anal. Appl. 433, 1718-1735 (2016)

9. Bandle, C., Brunner, H.: Blow-up in diffusion equations, a survey. J. Comput. Appl. Math. 97, 3-22 (1998)

10. Chung, S.-Y., Choi, M.-J.: A new condition for blow-up solutions to discrete semilinear heat equations on networks. Comput. Math. Appl. 74, 2929-2939 (2017)

11. Chung, S.Y., Choi, M.-J.: A new condition for the concavity method of blow-up solutions to p-Laplacian parabolic equations. J. Differ. Equ. 265(12), 6384-6399 (2018)

12. Chung, S.-Y., Hwang, J.: The discrete $p$-Schrödinger equations under the mixed boundary conditions on networks. Physica D (2019). https://doi.org/10.1016/j.physd.2019.02.009 
13. Chung, S.-Y.: Critical blow-up and global existence for discrete nonlinear p-Laplacian parabolic equations. Discrete Dyn. Nat. Soc. 10, Article ID 716327 (2014)

14. Chung, S.-Y., Hwang, J.: A complete characterization of the discrete p-Laplacian parabolic equations with q-nonlocal reaction with respect to the blow-up property. J. Math. Anal. Appl. 473(2), 1447-1473 (2019)

15. Li, J., Han, Y., Li, H.: Blow-up and extinction of solutions to a fast diffusion equation with homogeneous Neumann boundary conditions. Electron. J. Differ. Equ. 2016, 236 (2016)

16. Chung, S.-Y., Berenstein, C.A.: $\omega$-harmonic functions and inverse conductivity problems on network. SIAM J. Appl. Math. 65, 1200-1226 (2005)

17. Kim, J.-H., Chung, S.Y.: Comparison principles for the $p$-Laplacian on nonlinear networks. J. Differ. Equ. Appl. 16(10), $1151-1163(2010)$

18. Chung, F.R.K.: Spectral Graph Theory. CBMS Regional Conference Series in Math., vol. 92. Am. Math. Soc., Providence (1997)

19. Cvetkovic, D.M., Doob, M., Sachs, H.: Spectra of Graphs, Theory and Applications. Academic Press, New York (1980)

Submit your manuscript to a SpringerOpen ${ }^{\odot}$ journal and benefit from:

- Convenient online submission

- Rigorous peer review

- Open access: articles freely available online

- High visibility within the field

- Retaining the copyright to your article

Submit your next manuscript at $\boldsymbol{\nabla}$ springeropen.com 